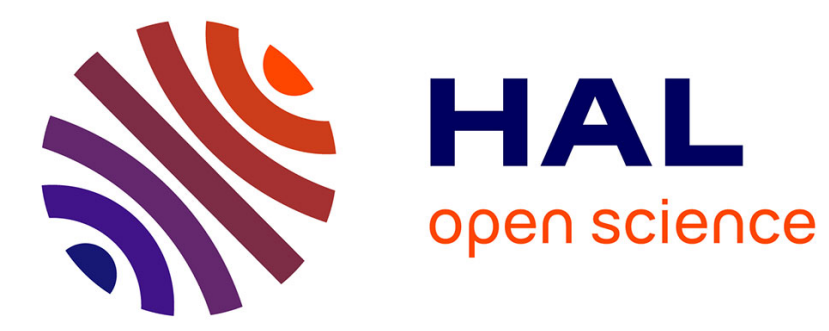

\title{
Robust synchronization for multistable systems
}

Hafiz Ahmed, Rosane Ushirobira, Denis Efimov, Wilfrid Perruquetti

\section{To cite this version:}

Hafiz Ahmed, Rosane Ushirobira, Denis Efimov, Wilfrid Perruquetti. Robust synchronization for multistable systems. IEEE Transactions on Automatic Control, 2016, 61 (6), pp.1625-1630. 10.1109/TAC.2015.2476156 . hal-01185112

\section{HAL Id: hal-01185112 \\ https://hal.inria.fr/hal-01185112}

Submitted on 21 Oct 2015

HAL is a multi-disciplinary open access archive for the deposit and dissemination of scientific research documents, whether they are published or not. The documents may come from teaching and research institutions in France or abroad, or from public or private research centers.
L'archive ouverte pluridisciplinaire HAL, est destinée au dépôt et à la diffusion de documents scientifiques de niveau recherche, publiés ou non, émanant des établissements d'enseignement et de recherche français ou étrangers, des laboratoires publics ou privés. 


\title{
Robust synchronization for multistable systems
}

\author{
Hafiz Ahmed, Rosane Ushirobira, Denis Efimov and Wilfrid Perruquetti
}

\begin{abstract}
In this note, we study a robust synchronization problem for multistable systems evolving on manifolds within an Input-to-State Stability (ISS) framework. Based on a recent generalization of the classical ISS theory to multistable systems, a robust synchronization protocol is designed with respect to a compact invariant set of the unperturbed system. The invariant set is assumed to admit a decomposition without cycles, that is, with neither homoclinic nor heteroclinic orbits. Numerical simulation examples illustrate our theoretical results.
\end{abstract}

\section{INTRODUCTION}

Over the last decades, the synchronization of complex dynamical systems and/or network of systems has attracted a great deal of attention from multidisciplinary research communities thanks to their pervasive presence in nature, technology and human society [1], [2], [3], [4]. A collective behavior occurs in the interconnection of dynamical systems and it has several potential application domains. For instance, transient stability in power network [5], cooperative multitasking and formation control [6]. The core of synchronization is the collective objective of agents in a network to reach a consensus about certain variables of interest.

The existing literature on the synchronization problem is very vast and covers many areas. In [7], the problem of formation control is investigated in swarms within the framework of output regulation in nonlinear systems. A detailed study regarding the control and synchronization of chaos can be found in [8]. The paper [9] extends optimal control and adaptive control design methods to multi-agent nonlinear systems on communication graphs. Recent advances in various aspects of cooperative control of multi-agent systems can be found in [10]. The theoretical framework for design and analysis of distributed flocking algorithms can be found in [11].

In this paper, we consider the synchronization problem for multistable systems based on the framework of Input-to-State Stability (ISS). This is a very well established method for the study of stability and robustness of nonlinear systems. The ISS property provides a natural framework of stability analysis with respect to input perturbations (see [12] and references therein). The classical definition allows to formulate and characterize stability properties with respect to arbitrary compact invariant sets (and not simply equilibria). Nevertheless, the implicit requirement that these sets should be simultaneously Lyapunov stable and

The authors are with the Non-A team @ Inria, 40 avenue Halley, 59650 Villeneuve d'Ascq, France. D. Efimov and W. Perruquetti are with CRIStAL UMR 9189, Ecole Centrale de Lille, Avenue Paul Langevin, 59651 Villeneuve d'Ascq, France. E-mail: \{hafiz.ahmed, rosane.ushirobira, denis.efimov, wilfrid.perruquetti@inria.fr\}

D. Efimov is with the Department of Control Systems and Informatics, University ITMO, 49 avenue Kronverkskiy, 197101 Saint Petersburg, Russia This work was supported in part by the Government of Russian Federation (Grant 074-U01) and the Ministry of Education and Science of Russian Federation (Project 14.Z50.31.0031).

H. Ahmed is partly supported by the regional council of Nord-Pas de Calais, France. globally attractive, makes the basic theory not applicable for a global analysis of many dynamical behaviors of interest, having multistability [13], [14], [15] or periodic oscillations [16], just to name a few, and only local analysis remains possible [17]. Some attempts were made to overcome such limitations by introducing the notions of almost global stability [18] and almost input-tostate stability [19], etc.

Recently, the authors in [20] have proposed that the most natural way of relaxing ISS condition for systems with multiple invariant sets is equivalent to relax the Lyapunov stability requirement [21] (rather than the global nature of the attractivity property). Using this relatively mild condition, they [20] have generalized the ISS theory as well as, the related literature on time invariant autonomous dynamical systems on compact spaces [22] for multistable systems. Multistability accounts for the possible coexistence of various oscillatory regimes or equilibria in the phase space of the system for the same set of parameters. Any system that exhibits multistability is called a multistable system. For a multistable system, it is frequently very difficult to predict the asymptotic regime on which this system will attain asymptotically for the given set of initial conditions and inputs [23]. In our current work, the results presented in [20] are applied to provide sufficient conditions for the existence of robust synchronization for multistable systems in the presence of external inputs. The conditions obtained in this work are global.

The rest of this paper is organized as follows. Section II introduces some preliminaries about decomposable sets and notions of robustness. Our main results and the family of nonlinear systems being considered can be found in Section III. In Section IV, numerical simulation examples are given to illustrate these results. Concluding remarks in Section V close this note.

\section{Preliminaries}

Let $M$ be an $n$-dimensional $\mathcal{C}^{2}$ connected and orientable Riemannian manifold without a boundary, $x \in M$ and $f$ : $M \times \mathbb{R}^{m} \rightarrow T_{x} M$ be a map of class $\mathcal{C}^{1}$. In this work, we assume that all manifolds are embedded in a Euclidean space of dimension $n$, so they contain 0 . Consider a nonlinear system of the following form:

$$
\dot{x}(t)=f(x(t), d(t))
$$

where the state $x(t) \in M$ and $d(t) \in \mathbb{R}^{m}$ (the input $d(\cdot)$ is a locally essentially bounded and measurable signal) for $t \geq 0$. We denote by $X(t, x ; d(\cdot))$ the uniquely defined solution of (1) at time $t$ satisfying $X(0, x ; d(\cdot))=x$. Together with (1), we will analyze its unperturbed version:

$$
\dot{x}(t)=f(x(t), 0) .
$$

A set $S \subset M$ is invariant for the unperturbed system (2) if $X(t, x ; 0) \in S$ for all $t \in \mathbb{R}$ and for all $x \in S$. For a set 
$S \subset M$, define the distance to $S$ from a point $x \in M$ by $|x|_{S}=\inf _{a \in S} \delta(x, a)$, where the $\delta\left(x_{1}, x_{2}\right)$ denotes the Riemannian distance between $x_{1}$ and $x_{2}$ in $M$. We have $|x|=|x|_{\{0\}}$ for $x \in M$, the usual Euclidean norm of a vector $x \in \mathbb{R}^{n}$. For a signal $d: \mathbb{R} \rightarrow \mathbb{R}^{m}$, the essential supremum norm is defined as $\|d\|_{\infty}=\operatorname{ess}_{\sup } \operatorname{su0}_{t \geq 0}|d(t)|$.

A function $\alpha: \mathbb{R}_{+} \rightarrow \mathbb{R}_{+}$is said to belong to class $\mathcal{K}$, i.e. $\alpha \in \mathcal{K}$, if it is continuous, strictly increasing and $\alpha(0)=0$. Furthermore, $\alpha \in \mathcal{K}_{\infty}$ if $\alpha \in \mathcal{K}$ and unbounded i.e. $\lim _{s \rightarrow \infty} \alpha(s)=\infty$. For any $x \in M$, the $\alpha$ - and $\omega$ - limit sets for (2) can be defined as follows:

$\alpha(x):=\left\{y \in M \mid y=\lim _{n \rightarrow-\infty} X\left(x, t_{n}\right)\right.$ with $\left.t_{n} \searrow-\infty\right\}$, $\omega(x):=\left\{y \in M \mid y=\lim _{n \rightarrow \infty} X\left(x, t_{n}\right)\right.$ with $\left.t_{n} \nearrow \infty\right\}$.

\section{A. Decomposable sets}

Let $\Lambda \subset M$ be a compact invariant set for (2).

Definition 1. [22] A decomposition of $\Lambda$ is a finite and disjoint family of compact invariant sets $\Lambda_{1}, \ldots, \Lambda_{k}$ such that $\Lambda=$ $\bigcup_{i=1}^{k} \Lambda_{i}$.

For an invariant set $\Lambda$, its attracting and repulsing subsets are defined as follows:

$$
\begin{aligned}
W^{s}(\Lambda) & =\left\{\left.x \in M|| X(t, x, 0)\right|_{\Lambda} \rightarrow 0 \text { as } t \rightarrow+\infty\right\} \\
W^{u}(\Lambda) & =\left\{\left.x \in M|| X(t, x, 0)\right|_{\Lambda} \rightarrow 0 \text { as } t \rightarrow-\infty\right\} .
\end{aligned}
$$

Define a relation on the set of invariant sets of $M$ by: for $\mathcal{W} \subset M$ and $\mathcal{D} \subset M$, we write $\mathcal{W} \prec \mathcal{D}$ if $W^{s}(\mathcal{W}) \cap W^{u}(\mathcal{D}) \neq \emptyset$.

Definition 2. [22] Let $\Lambda_{1}, \ldots, \Lambda_{k}$ be a decomposition of $\Lambda$, then

1) An $r$-cycle $(r \geq 2)$ is an ordered $r$-tuple of distinct indices $i_{1}, \ldots, i_{r}$ such that $\Lambda_{i_{1}} \prec \ldots \prec \Lambda_{i_{r}} \prec \Lambda_{i_{1}}$.

2) A 1-cycle is an index $i$ such that $\left(W^{u}\left(\Lambda_{i}\right) \cap W^{s}\left(\Lambda_{i}\right)\right) \backslash$ $\Lambda_{i} \neq \emptyset$.

3) A filtration ordering is a numbering of the $\Lambda_{i}$ so that $\Lambda_{i} \prec$ $\Lambda_{j} \Rightarrow i \leq j$.

As we can conclude from Definition 2, the existence of an $r$ cycle with $r \geq 2$ is equivalent to the existence of a heteroclinic cycle for (2) [24]. Moreover, the existence of a 1-cycle implies the existence of a homoclinic cycle for (2) [24].

Definition 3. Let $\mathcal{W} \subset M$ be a compact set containing all $\alpha$ and $\omega$ limit sets of (2). We say that $\mathcal{W}$ is decomposable if it admits a finite decomposition without cycles, $\mathcal{W}=\bigcup_{i=1}^{k} \mathcal{W}_{i}$, for some non-empty disjoint compact sets $\mathcal{W}_{i}$, forming a filtration ordering of $\mathcal{W}$. This definition of the compact set $\mathcal{W}$ will be used all through the article.

\section{B. Robustness notions}

The following robustness notions for systems in (1) have been introduced in [20].

Definition 4. We say that the system (1) has the practical asymptotic gain (pAG) property if there exist $\eta \in \mathcal{K}_{\infty}$ and $q \in \mathbb{R}$, $q \geq 0$ such that for all $x \in M$ and all measurable essentially bounded inputs $d(\cdot)$, the solutions are defined for all $t \geq 0$ and

$$
\limsup _{t \rightarrow+\infty}|X(t, x ; d)|_{\mathcal{W}} \leq \eta\left(\|d\|_{\infty}\right)+q \text {. }
$$

If $q=0$, then we say that the asymptotic gain (AG) property holds.

Definition 5. We say that the system (1) has the limit property (LIM) with respect to $\mathcal{W}$ if there exists $\mu \in \mathcal{K}_{\infty}$ such that for all $x \in M$ and all measurable essentially bounded inputs $d(\cdot)$, the solutions are defined for all $t \geq 0$ and the following holds:

$$
\inf _{t \geq 0}|X(t, x ; d)| \mathcal{W} \leq \mu\left(\|d\|_{\infty}\right)
$$

Definition 6. We say that the system (1) has the practical global stability (pGS) property with respect to $\mathcal{W}$ if there exist $\beta \in \mathcal{K}_{\infty}$ and $q \geq 0$ such that for all $x \in M$ and all measurable essentially bounded inputs $d(\cdot)$, the following holds for all $t \geq 0$ :

$$
|X(t, x ; d)|_{\mathcal{W}} \leq q+\beta\left(\max \left\{|x|_{\mathcal{W}},\|d\|_{\infty}\right\}\right) .
$$

To characterize (3) in terms of Lyapunov functions, it has been shown in [20] that the following notion suits:

Definition 7. We say that a $\mathcal{C}^{1}$ function $V: M \rightarrow \mathbb{R}$ is a practical ISS-Lyapunov function for (1) if there exists $\mathcal{K}_{\infty}$ functions $\alpha_{1},\left[\alpha_{2}\right], \alpha$ and $\gamma$, and scalar $q \geq 0$ [and $c \geq 0$ ] such that

$$
\alpha_{1}\left(|x|_{\mathcal{W}}\right) \leq V(x) \leq\left[\alpha_{2}\left(|x|_{\mathcal{W}}+c\right)\right],
$$

the function $V$ is constant on each $\mathcal{W}_{i}$ and the dissipation inequality below holds:

$$
D V(x) f(x, d) \leq-\alpha\left(|x|_{\mathcal{W}}\right)+\gamma(|d|)+q .
$$

If this latter holds for $q=0$, then $V$ is said to be an ISSLyapunov function.

Notice that $\alpha_{2}$ and $c$ are in square brackets as their existence follows (without any additional assumptions) by standard continuity arguments.

The main result of [20] connecting these robust stability properties is stated below:

Theorem 8. Consider a nonlinear system as in (1) and let a compact invariant set containing all $\alpha$ and $\omega$ limit sets of (2) $\mathcal{W}$ be decomposable (in the sense of Definition 3). Then the following are equivalent:

1) The system admits an ISS Lyapunov function;

2) The system enjoys the AG property;

3) The system admits a practical ISS Lyapunov function;

4) The system enjoys the $p A G$ property;

5) The system enjoys the LIM property and the pGS.

A system in (1) that satisfies this list of equivalent properties is called ISS with respect to the set $\mathcal{W}$ [20].

\section{SYNCHRONIZATION OF MULTISTABLE SYSTEMS}

The following family of nonlinear systems is considered in this section:

$$
\dot{x}_{i}(t)=f_{i}\left(x_{i}(t), u_{i}(t), d_{i}(t)\right), i=1, \ldots, N, N>1,
$$

where the state $x_{i}(t) \in M_{i}$, with $M_{i}$ an $n_{i}$-dimensional $\mathcal{C}^{2}$ connected and orientable Riemannian manifold without a boundary, the control $u_{i}(t) \in \mathbb{R}^{m_{i}}$ and the external disturbance $d_{i}(t) \in \mathbb{R}^{p_{i}}$ $\left(u_{i}(\cdot)\right.$ and $d_{i}(\cdot)$ are locally essentially bounded and measurable signals) for $t \geq 0$ and the map $f_{i}: M_{i} \times \mathbb{R}^{m_{i}} \times \mathbb{R}^{p_{i}} \rightarrow T_{x_{i}} M_{i}$ 
is $\mathcal{C}^{1}, f_{i}(0,0,0)=0$. Denote the common state vector of (4) as $x=\left[x_{1}^{T}, \ldots, x_{N}^{T}\right]^{T} \in M=\prod_{i=1}^{N} M_{i}$, so $M$ is the corresponding Riemannian manifold of dimension $n=\sum_{i=1}^{N} n_{i}$ where the family (4) behaves and $d=\left[d_{1}^{T}, \ldots, d_{N}^{T}\right]^{T} \in \mathbb{R}^{p}$ with $p=\sum_{i=1}^{N} p_{i}$ is the total exogenous input.

Assumption 1. For all $i=1, \ldots, N$, each system in (4) has a compact invariant set $\mathcal{W}_{i}$ containing all $\alpha$ and $\omega$ limit sets of $\dot{x}_{i}(t)=f_{i}\left(x_{i}(t), 0,0\right), \mathcal{W}_{i}$ is decomposable in the sense of Definition 3, and the system enjoys the AG property with respect to inputs $u_{i}$ and $d_{i}$ as in Definition 4.

Under this assumption, from Theorem 8 , there exist $\mathcal{C}^{1}$ ISS-Lyapunov functions $V_{i}: M_{i} \rightarrow \mathbb{R}$ with $\mathcal{K}_{\infty}$ functions $\alpha_{1 i}, \alpha_{2 i}, \alpha_{3 i}, \gamma_{u i}$ and $\gamma_{d i}$ such that

$$
\alpha_{1 i}\left(\left|x_{i}\right| \mathcal{W}_{i}\right) \leq V_{i}\left(x_{i}\right) \leq \alpha_{2 i}\left(\left|x_{i}\right|_{\mathcal{W}_{i}}+c_{i}\right), c_{i} \geq 0
$$

$D V_{i}\left(x_{i}\right) f_{i}\left(x_{i}, u_{i}, d_{i}\right) \leq-\alpha_{3 i}\left(\left|x_{i}\right| \mathcal{W}_{i}\right)+\gamma_{u i}\left(\left|u_{i}\right|\right)+\gamma_{d i}\left(\left|d_{i}\right|\right)$

for all $i=1, \ldots, N$. Define also the invariant set of disconnected and unperturbed $\left(u_{i}=d_{i}=0\right)$ family $\mathcal{W}=\prod_{i=1}^{N} \mathcal{W}_{i} \subset M$ $(0 \in \mathcal{W})$. Then, by definition, there exist functions $\nu_{1}, \nu_{2} \in \mathcal{K}_{\infty}$ such that

$$
\nu_{1}\left(|x|_{\mathcal{W}}\right) \leq \sum_{i=1}^{N}\left|x_{i}\right|_{\mathcal{W}_{i}} \leq \nu_{2}\left(|x|_{\mathcal{W}}\right)
$$

for all $x \in M$. Since the set $\mathcal{W}$ is compact, then there are functions $\nu_{3}, \nu_{4} \in \mathcal{K}_{\infty}$ and a scalar $c_{0} \geq 0$ such that for all $x \in M$,

$$
|x| \leq \nu_{3}\left(|x|_{\mathcal{W}}\right)+c_{0},|x|_{\mathcal{W}} \leq \nu_{4}(|x|)
$$

Hence, we will consider in this work, the family (4) under Assumption 1, i.e. a family of robustly stable nonlinear systems. In general, the sets $\mathcal{W}_{i}$ include equilibrium (at the origin, for instance) and limit cycles of agents in (4). There are several works devoted to synchronization and design of consensus protocols for such a family or oscillatory network [25], [26], [27]. The goal of our study is to find a condition under which the existence of a global synchronization/consensus protocol for $d=0$ implies robust synchronization in (4) for a bounded $d \neq 0$.

Let a $\mathcal{C}^{1}$ function $y(x): M \rightarrow \mathbb{R}^{q}, y(0)=0$ be a synchronization measure for (4). We say that the family (4) is synchronized (or reached the consensus) if $y(x(t)) \equiv 0$ for all $t \geq 0$ on the solutions of the network under properly designed control actions

$$
u_{i}(t)=\varphi_{i}[y(x(t))]
$$

$\left(\varphi_{i}: \mathbb{R}^{q} \rightarrow \mathbb{R}^{m_{i}}\right.$ is a $\mathcal{C}^{1}$ function, $\left.\varphi_{i}(0)=0\right)$ for $d(t) \equiv 0$, $t \geq 0$. In this case the set $\mathcal{A}=\{x \in \mathcal{W} \mid y(x)=0\}$ contains the synchronous solutions of the unperturbed family in (4) and the problem of synchronization of "natural" trajectories is considered since $\mathcal{A} \subset \mathcal{W}$. Due to the condition $\varphi_{i}(0)=0$, the convergence of $y$ (synchronization/consensus) implies that the solutions of the interconnection belong to $\mathcal{W}$, the conditions of convergence of the synchronizing/consensus output $y$ can be found in [25], [26], [27].

The proposed synchronization protocol is output based, as in [25], [26], [27]. The synchronization measure $y$ in general depends on some elements of the vectors $x_{i}$ for all $i=1, \ldots, N$.
In addition, since $y$ is a vector, then different topology of interconnection can be imposed, see examples in Section IV.

Assumption 2. The set $\mathcal{A}$ is compact, it contains all $\alpha$ and $\omega$ limit sets of (4), (8) for $d=0$, and it is decomposable.

Therefore, it is assumed that the controls $\varphi_{i}(y)$ ensure the network global synchronization, while decomposability in general follows from Assumption 1. We will show that in the setup as above, by selecting the shapes of $\varphi_{i}$, it is possible to guarantee robust synchronization of (4) for any measurable and essentially bounded input $d$.

By continuity arguments, there exist functions $\eta_{1}, \eta_{2}, \mu_{i} \in$ $\mathcal{K}_{\infty}$ with a scalar $\eta_{0} \geq 0$ such that for all $x \in M$ :

$$
\begin{gathered}
|y(x)| \leq \eta_{0}+\eta_{1}(|x| \mathcal{W}),|y(x)| \leq \eta_{2}(|x|), \\
\left|\varphi_{i}(y)\right| \leq \mu_{i}(|y|)
\end{gathered}
$$

(note that the first two inequalities are related through (7)). Then the intermediate result below can be proven under Assumption 1 for (4), (8).

Proposition 9. Let Assumption 1 be satisfied for (4). Then there exist $\varphi_{i}, i=1, \ldots, N$ in (8) such that the interconnection (4), (8) has pGS property with respect to the set $\mathcal{W}$.

Proof. Consider a Lyapunov function candidate $S(x)=$ $\sum_{i=1}^{N} V_{i}\left(x_{i}\right)$, where the functions $V_{i}$ are given in (5). From (6), there exist two functions $\underline{\alpha}, \bar{\alpha} \in \mathcal{K}_{\infty}$ and a scalar $g \geq 0$ such that for all $x \in M$ :

$$
\underline{\alpha}\left(|x|_{\mathcal{W}}\right) \leq S(x) \leq \bar{\alpha}\left(|x|_{\mathcal{W}}+g\right) .
$$

Taking the derivative of $S$ with respect to equations in (4), (8) we obtain:

$$
\dot{S} \leq \sum_{i=1}^{N}\left[-\alpha_{3 i}\left(\left|x_{i}\right| \mathcal{W}_{i}\right)+\gamma_{u i}\left(\left|\varphi_{i}(y)\right|\right)+\gamma_{d i}\left(\left|d_{i}\right|\right)\right]
$$

From (6) and (9), we deduce:

$$
\begin{aligned}
\sum_{i=1}^{N} \alpha_{3 i}\left(\left|x_{i}\right| \mathcal{W}_{i}\right) & \geq 2 \alpha_{4}\left(|x|_{\mathcal{W}}\right) \\
\sum_{i=1}^{N} \gamma_{u i}\left(\left|\varphi_{i}(y)\right|\right) & \leq \sum_{i=1}^{N} \gamma_{u i} \circ \mu_{i}(|y|) \\
& \leq \sum_{i=1}^{N} \gamma_{u i} \circ \mu_{i}\left(\eta_{0}+\eta_{1}\left(|x|_{\mathcal{W}}\right)\right) \\
& \leq h+\sum_{i=1}^{N} \gamma_{u i} \circ \mu_{i}\left(2 \eta_{1}\left(|x|_{\mathcal{W}}\right)\right)
\end{aligned}
$$

for some $\alpha_{4} \in \mathcal{K}_{\infty}$ and where $h=\sum_{i=1}^{N} \gamma_{u i} \circ \mu_{i}\left(2 \eta_{0}\right)$. By optimizing the shape of $\varphi_{i}$, it is possible to adjust the form of $\mu_{i}$. In particular, providing that

$$
\mu_{i}(s) \leq \gamma_{u i}^{-1}\left[N^{-1} \alpha_{4} \circ \eta_{1}^{-1}(0.5 s)\right]
$$

for all $i=1, \ldots, N$ we guarantee the relation $\gamma_{u i} \circ \mu_{i}\left(2 \eta_{1}(s)\right) \leq$ $\frac{1}{N} \alpha_{4}(s)$, then

$$
\sum_{i=1}^{N} \gamma_{u i}\left(\left|\varphi_{i}(y)\right|\right) \leq h+\sum_{i=1}^{N} \frac{1}{N} \alpha_{4}(s) \leq h+\alpha_{4}(s) .
$$


Substituting the obtained terms in the inequality derived for $\dot{S}$, we obtain

$$
\dot{S} \leq-\alpha_{4}\left(|x|_{\mathcal{W}}\right)+h+\gamma_{d}(|d|),
$$

where $\gamma_{d}$ is a function from class $\mathcal{K}_{\infty}$ such that $\sum_{i=1}^{N} \gamma_{d i}\left(\left|d_{i}\right|\right) \leq \gamma_{d}(|d|)$. Finally, $\alpha_{4} \circ \bar{\alpha}^{-1}[S(x)] \leq$ $\alpha_{4}\left(2|x|_{\mathcal{W}}\right)+\alpha_{4}(2 g)$ and

$$
\dot{S} \leq-\alpha_{4} \circ \bar{\alpha}^{-1}(S)+h+\alpha_{4}(2 g)+\gamma_{d}(|d|),
$$

which by the standard arguments [28] implies that for all $t \geq 0$

$$
S(t) \leq \beta(S(0), t)+r+\gamma_{d}^{\prime}\left(\|d\|_{\infty}\right)
$$

for some function $\beta \in \mathcal{K} \mathcal{L}, \gamma_{d}^{\prime} \in \mathcal{K}$ and a scalar $r \geq 0$. The pGS property follows taking in mind that $\underline{\alpha}\left(|x(t)|_{\mathcal{W}}\right) \leq S(t)$, $S(0) \leq[\bar{\alpha}(|x(0)| \mathcal{W}+g)]$ and the properties of a function from the class $\mathcal{K} \mathcal{L}$.

Note that by definition of the set $\mathcal{A},|x(t)|_{\mathcal{W}} \leq|x(t)|_{\mathcal{A}} \leq$ $|x(t)|_{\mathcal{W}}+z$ for a scalar $z \geq 0$ for all $x \in M$, then the pGS property with respect to the set $\mathcal{A}$ has also been proven.

Therefore, in the setup used in this work the boundedness of trajectories (boundedness of $|x(t)| \mathcal{W}$ implies the same property for $|x(t)|$ according to (7)) follows by a proper selection of the interconnection gain in (8), i.e. by decreasing the control gain a certain robustness of (4), (8) is inherited after individual systems as it is stated in Assumption 1.

Theorem 10. Let assumptions 1 and 2 be satisfied for (4), (8). Then there exist $\varphi_{i}, i=1, \ldots, N$ in (8) such that the interconnection (4), (8) has AG property with respect to $\mathcal{A}$.

Proof. Since all conditions of Proposition 9 are satisfied, by a proper selection of $\varphi_{i}$, the Lyapunov function $S$ has the properties as in the proof above. From (9) $\alpha_{4} \circ \eta_{1}^{-1}(0.5|y(x)|) \leq \alpha_{4} \circ$ $\eta_{1}^{-1}\left(\eta_{0}\right)+\alpha_{4}\left(|x|_{\mathcal{W}}\right)$. Then

$$
\dot{S} \leq-0.5 \alpha_{4}\left(|x|_{\mathcal{W}}\right)-0.5 \alpha_{5}(|y(x)|)+h^{\prime}+\gamma_{d}(|d|),
$$

where $h^{\prime}=h+0.5 \alpha_{4} \circ \eta_{1}^{-1}\left(\eta_{0}\right)$ and $\alpha_{5}(s)=\alpha_{4} \circ \eta_{1}^{-1}(0.5 s)$. By the definition of the set $\mathcal{A}$, there exists $\theta \in \mathcal{K}_{\infty}$ such that $\alpha_{4}\left(|x|_{\mathcal{W}}\right)+\alpha_{5}(|y(x)|) \geq 2 \theta\left(|x|_{\mathcal{A}}\right)$ for all $x \in M$, then

$$
\dot{S} \leq-\theta\left(|x|_{\mathcal{A}}\right)+h^{\prime}+\gamma_{d}(|d|) .
$$

According to Proposition 9, the solutions are bounded. Hence, the system (4), (8) is forward complete. Following [29], for any forward complete system, there exists a smooth function $Q$ : $M \rightarrow \mathbb{R}$ (the proof in [29] deals with Euclidean spaces, but similar arguments can be adopted here) such that for all $x \in M$ and $d \in \mathbb{R}^{p}$

$$
\psi_{1}(|x|) \leq Q(x) \leq \psi_{2}(|x|), \dot{Q} \leq 1+\rho(|d|)
$$

for some $\psi_{1}, \psi_{2}, \rho \in \mathcal{K}_{\infty}$. Note that there exists $\nu_{5} \in \mathcal{K}_{\infty}$ such that $|x|_{\mathcal{A}} \leq \nu_{5}(|x|)$ for all $x \in M$ similarly to (7). Let us introduce a practical ISS Lyapunov function $U(x)=Q(x)+$ $S(x)$ for (4), (8), then for all $x \in M$ and $d \in \mathbb{R}^{p}$ we have

$$
\begin{gathered}
\underline{\alpha}^{\prime}\left(|x|_{\mathcal{A}}\right) \leq U(x) \leq \bar{\alpha}^{\prime}\left(|x|_{\mathcal{A}}+g^{\prime}\right), \\
\dot{U} \leq-\theta\left(|x|_{A}\right)+h^{\prime}+1+\gamma_{d}(|d|)+\rho(|d|)
\end{gathered}
$$

for properly defined $\underline{\alpha}^{\prime}, \bar{\alpha}^{\prime} \in \mathcal{K}_{\infty}$ and a scalar $g^{\prime} \geq 0$. Thus, $U$ admits all requirements imposed on practical ISS Lyapunov functions, and under Assumption 2 the system (4), (8) possesses all properties in Theorem 8 and it is ISS with respect to $\mathcal{A}$.

Roughly speaking, this qualitative result states that if the synchronized output $y$ is related with $|x|_{\mathcal{W}}$ as in (9) and each system in the network is robustly stable as in Assumption 1, then the system can be robustly synchronized by a sufficiently small feedback proportional to $y$.

\section{EXAMPLES AND SIMULATIONS}

\section{A. Application to nonlinear pendulums without friction}

Consider a network of nonlinear identical pendulums for $i=$ $1, \ldots, N, N>1$ :

$$
\begin{aligned}
& \dot{x}_{1 i}=x_{2 i}, \\
& \dot{x}_{2 i}=-\omega \sin \left(x_{1 i}\right)+v_{i}+d_{i},
\end{aligned}
$$

where the state $x_{i}=\left[x_{1 i}, x_{2 i}\right]$ takes values on the cylinder $M_{i}:=\mathbb{S} \times \mathbb{R}$, the exogenous disturbance $d_{i}(t) \in \mathbb{R}$, the regulation input $u_{i}(t) \in \mathbb{R}$, and $\omega$ is a constant positive parameter. The unperturbed system is conservative with Hamiltonian $H\left(x_{i}\right)=0.5 x_{2 i}^{2}+\omega\left(1-\cos \left(x_{1 i}\right)\right)$ and $\dot{H}=x_{2 i}\left(v_{i}+d_{i}\right)$. The control $v_{i}$ will have two parts, one to force controlled oscillations in (10) and one for the synchronization $u_{i}$ :

$$
v_{i}=-x_{2 i}\left[H\left(x_{i}\right)-H^{*}\right]+u_{i},
$$

where $0<H^{*}<2 \omega$ is the desired level of $H\left(x_{i}\right)$ that defines the attracting limit cycle $\Gamma_{i}=\left\{x \in M_{i}: H\left(x_{i}\right)=H^{*}\right\}$ in

$$
\begin{aligned}
& \dot{x}_{1 i}=x_{2 i} \\
& \dot{x}_{2 i}=-\omega \sin \left(x_{1 i}\right)-x_{2 i}\left[H\left(x_{i}\right)-H^{*}\right]+u_{i}+d_{i} .
\end{aligned}
$$

Despite of the limit cycle $\Gamma_{i}$, each unperturbed system admits also two equilibria $[0,0]$ and $[\pi, 0]$, the latter being a saddle point. Thus $\mathcal{W}_{i}=\left\{[0,0] \cup[\pi, 0] \cup \Gamma_{i}\right\}$. Clearly, $\mathcal{W}_{i}$ is compact and contains all $\alpha$ and $\omega$ limit sets of (11) for $u_{i}=d_{i}=0$. Moreover, it is straightforward to check that $\mathcal{W}_{i}$ is decomposable in the sense of Definition 3.

Lemma 11. For each $i=1, \ldots, N$, the systems in (11) have $A G$ property.

Proof. The conditions of Theorem 8 are satisfied for the system (11) and $\mathcal{W}_{i}$, thus it is enough to check a practical AG in this case. First, $\left|x_{1 i}(t)\right| \leq \pi$ for all $t \geq 0$ by definition, and it is necessary to show a pAG for the coordinate $x_{2 i}$ only. For this purpose, we consider $W\left(x_{2 i}\right)=0.5 x_{2 i}^{2}$. Hence:

$$
\begin{aligned}
\dot{W}= & x_{2 i}\left[-\omega \sin \left(x_{1 i}\right)-x_{2 i}\left[H\left(x_{i}\right)-H^{*}\right]+u_{i}+d_{i}\right] \\
= & x_{2 i}\left[-\omega \sin \left(x_{1 i}\right)-x_{2 i}\left[0.5 x_{2 i}^{2}+\omega\left(1-\cos \left(x_{1 i}\right)\right)\right.\right. \\
& \left.\left.-H^{*}\right]+u_{i}+d_{i}\right] \\
\leq & \omega\left|x_{2 i}\right|-x_{2 i}^{2}\left[0.5 x_{2 i}^{2}+\omega\left(1-\cos \left(x_{1 i}\right)\right)\right. \\
& \left.-H^{*}\right]+0.5 x_{2 i}^{2}+0.5\left(u_{i}+d_{i}\right)^{2} \\
\leq & -0.5 x_{2 i}^{4}+\left(0.5+H^{*}+2 \omega\right) x_{2 i}^{2}+\omega\left|x_{2 i}\right| \\
& +0.5\left(u_{i}+d_{i}\right)^{2} .
\end{aligned}
$$

Since $0.5+H^{*}+2 \omega>0$ and $\omega>0$, there exists $f_{\max }>0$ such that $-0.25 x_{2 i}^{4}+\left(0.5+H^{*}+2 \omega\right) x_{2 i}^{2}+\omega\left|x_{2 i}\right| \leq f_{\max }$ for 
all $x_{2 i} \in \mathbb{R}$, then

$$
\begin{aligned}
\dot{W} & \leq-0.25 x_{2 i}^{4}+f_{\max }+0.5\left(u_{i}+d_{i}\right)^{2} \\
& \leq-W^{2}+f_{\max }+0.5\left(u_{i}+d_{i}\right)^{2} .
\end{aligned}
$$

Next, applying standard arguments:

$$
\begin{aligned}
& \limsup _{t \rightarrow+\infty} W(t) \leq \sqrt{f_{\max }+0.5\left(\left\|u_{i}\right\|_{\infty}+\left\|d_{i}\right\|_{\infty}\right)^{2}} \text { and } \\
& \limsup _{t \rightarrow+\infty}\left|x_{2 i}(t)\right|^{2} \leq 2 \sqrt{f_{\max }+0.5\left(\left\|u_{i}\right\|_{\infty}+\left\|d_{i}\right\|_{\infty}\right)^{2}} .
\end{aligned}
$$

Take $\left|x_{i}\right|=\sqrt{x_{1 i}^{2}+x_{2 i}^{2}}$ then

$$
\limsup _{t \rightarrow+\infty}\left|x_{i}(t)\right| \leq \sqrt{\pi^{2}+2 \sqrt{f_{\text {max }}+0.5\left(\left\|u_{i}\right\|_{\infty}+\left\|d_{i}\right\|_{\infty}\right)^{2}}}
$$

and the pAG property holds since $\left|x_{i}\right|_{\mathcal{W}_{i}} \leq\left|x_{i}\right|$.

As a consequence, Assumption 1 is satisfied for (11) and we may select the synchronization measure $y$ for the network. The synchronization problem for nonlinear pendulums has been widely considered previously [30], [31], [26] (usually for unperturbed systems without a limit cycle, for example, with $v_{i}=-\kappa x_{2 i}+u_{i}$ for some $\kappa>0$ ). In this work we will consider

$$
y=A x_{2},
$$

where $x_{2}=\left[x_{21}, \ldots, x_{2 N}\right]^{T}$ and $A \in \mathbb{R}^{N \times N}$ is a Metzler matrix whose off-diagonal elements $A_{i, j} \in\{0,1\}$ for all $1 \leq$ $i \neq j \leq N$ and $\sum_{j=1}^{N} A_{i j}=0, \sum_{j=1}^{N}\left|A_{i j}\right| \neq 0$ for all $i=$ $1, \ldots, N$ (for example, $A=\left[\begin{array}{cc}-1 & 1 \\ 1 & -1\end{array}\right]$ for $N=2$ ). It is necessary to check (9) for this $y$ : obviously the function $\eta_{2}$ exists. To evaluate the constant $\eta_{0}$ and the function $\eta_{1}$ it is necessary to calculate $\left|x_{i}\right|_{\mathcal{W}_{i}}$ (and $\left|x_{i}\right| \mathcal{W}$ ). Note that $|y|^{2} \leq\|A\|_{2} \sum_{j=1}^{N} x_{2 i}^{2}$, then it is enough to estimate a relation between $x_{2 i}$ and $\left|x_{i}\right|_{\mathcal{W}_{i}}$. There exist $\delta_{1}, \delta_{2} \in \mathcal{K}_{\infty}$ such that for all $x \in M_{i}$

$$
\delta_{1}\left(\left|x_{i}\right|_{\mathcal{W}_{i}}\right) \leq \Delta\left(x_{i}\right) \leq \delta_{2}\left(\left|x_{i}\right|_{\mathcal{W}_{i}}\right)
$$

where $\Delta\left(x_{i}\right)=\min \left\{\sin ^{2}\left(x_{1 i}\right)+0.5 x_{2 i}^{2},\left|H\left(x_{i}\right)-H^{*}\right|\right\}$. Then it is enough to establish the boundedness of $x_{2 i}$ by $\Delta\left(x_{i}\right)$, but a direct computation shows:

$$
0.5 x_{2 i}^{2} \leq \Delta\left(x_{i}\right)+H^{*}
$$

and (9) is valid for $y$. Take

$$
\varphi_{i}(y)=\epsilon \tanh \left(y_{i}\right), \epsilon>0,
$$

then we may suppose that Assumption 2 is satisfied for some sufficiently small $\epsilon$. The results of simulations confirm this conclusion, see for example Fig. 1, where for $N=4$ and

$$
A=\left[\begin{array}{cccc}
-2 & 1 & 1 & 0 \\
0 & -2 & 1 & 1 \\
1 & 0 & -2 & 1 \\
1 & 1 & 0 & -2
\end{array}\right], \omega=2, H^{*}=2, \epsilon=0.1
$$

the results for two scenarios are given: Fig. 1,a without disturbances and Fig. 1,b with disturbances as $\left[d_{1}, d_{2}, d_{3}, d_{4}\right]^{T}=$ $[0.7 \sin (2 t),-0.25 \sin (0.5 t),-0.8 \sin (10 t), \sin (25 t)]^{T}$.
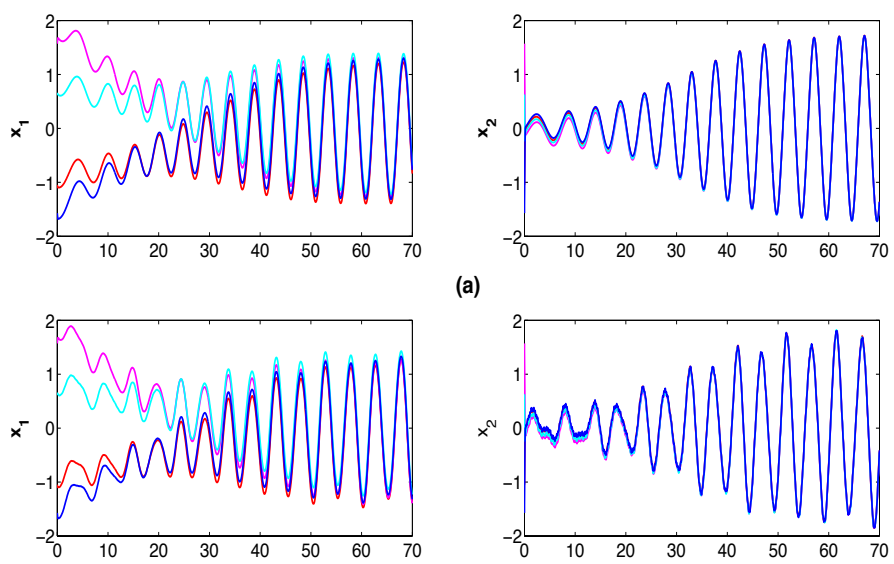

Figure 1. The results of simulation for (11)

\section{B. Application to nonlinear pendulums with friction}

This example is taken from [26]. Consider a network of nonlinear non-identical pendulums for $i=1, \ldots, N, N>1$ :

$$
\begin{aligned}
& \dot{x}_{1 i}=x_{2 i}, \\
& \dot{x}_{2 i}=-\Omega_{i}^{2} \sin \left(x_{1 i}\right)-\kappa x_{2 i}+d_{i},
\end{aligned}
$$

where the state $x_{i}=\left[x_{1 i}, x_{2 i}\right]$ takes values on the cylinder $M_{i}:=\mathbb{S} \times \mathbb{R}$, the exogenous disturbance $d_{i}(t) \in \mathbb{R}, \kappa$ is a constant parameter and $\Omega_{i}^{2}$ is the angular frequency of individual pendulums. The unperturbed system has a Hamiltonian $H\left(x_{i}\right)=0.5 x_{2 i}^{2}+\Omega_{i}^{2}\left(1-\cos \left(x_{1 i}\right)\right)$ and $\dot{H}=x_{2 i} d_{i}-\kappa x_{2 i}^{2}$. Each unperturbed system has two equilibria $[0,0]$ and $[\pi, 0]$ (the former is attractive and the later one is a saddle-point), thus $\mathcal{W}_{i}=\{[0,0] \cup[\pi, 0]\}$ is a compact set containing all $\alpha$ - and $\omega$-limit sets of (12) for $d_{i}=0$. In addition, it is easy to check that $\mathcal{W}_{i}$ is decomposable in the sense of Definition 3 [32].

Lemma 12. [33] For each $i=1, \ldots, N$, the systems in (12) is ISS with respect to the set $\mathcal{W}_{i}$.

As a consequence, Assumption 1 is satisfied for (12) (remark that admitting an ISS Lyapunov function is equivalent to enjoying AG property by Theorem 8 ) and we may select the synchronization measure $y$ for the network. Since in [26], the authors have considered the first coordinate as synchronization measure, we follow here the same idea:

$$
y=A \sin \left(x_{1}\right),
$$

where $x_{1}=\left[x_{11}, \ldots, x_{1 N}\right]^{T}$ and $A \in \mathbb{R}^{N \times N}$ is a Metzler matrix as in the first example.

Since the global boundedness of trajectories of (12) for bounded inputs is proven in Lemma 12, then a local analysis around equilibria is sufficient to show the synchronization measure convergence. It is straightforward to check that linearized around equilibria dynamics has $y=0$ as a stable and attractive manifold. By this, the convergence of $y$ is guaranteed locally. Then by taking,

$$
\varphi_{i}(y)=\beta y_{i}, \beta>0,
$$

we may suppose that Assumption 2 is satisfied for some sufficiently small $\beta$. The results of simulations confirm this 

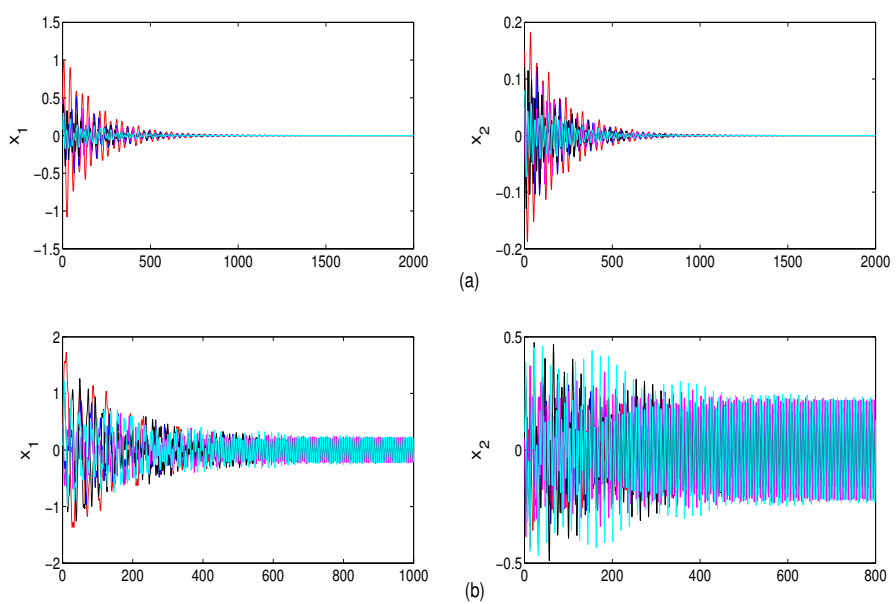

Figure 2. The result of simulation for (12)

conclusion, see Fig. 2 where a) is the disturbance free case and $b$ ) represents the simulation result with disturbances. The simulation parameters are $N=5, \Omega_{i}^{2}=0.02 i, \beta=$ 0.1 , the disturbance inputs are $\left[\phi_{1}, \ldots, \phi_{5}\right]^{T}=[0.1 \sin (t)$, $-0.15 \sin (t),-0.2 \sin (t), 0.15 \sin (t), 0.2 \sin (t)]^{T}$ and

$$
A=\left[\begin{array}{ccccc}
-3 & 1 & 1 & 0 & 1 \\
1 & -3 & 1 & 1 & 0 \\
1 & 1 & -3 & 1 & 0 \\
0 & 1 & 1 & -3 & 1 \\
1 & 0 & 0 & 1 & -2
\end{array}\right]
$$

\section{CONCLUSIONS}

In this work, sufficient conditions for robust synchronization were derived based on an extension of the ISS framework to systems evolving on a (non-compact) manifold and with multiple invariant sets. The condition imposed on the controller $\left(\varphi_{i}(0)=\right.$ 0 ) ensures that the convergence of the synchronization measure implies that the interconnection belongs to the decomposable set $\mathcal{W}$. Practical global stability analysis of the interconnection was done with respect to $\mathcal{W}$. The asymptotic gain property of the interconnection with respect to the set of synchronous solutions $\mathcal{A}(\mathcal{A} \subset \mathcal{W})$ was also proved.

Numerical simulations demonstrated the effectiveness of our method to network of both identical and nonidentical nodes. Remark that our results are applicable only to systems that allow decomposition without cycles.

\section{REFERENCES}

[1] I. I. Blekhman, Synchronization in science and technology. ASME Press, 1988.

[2] G. V. Osipov, J. Kurths, and C. Zhou, Synchronization in Oscillatory Networks. Springer, 2007.

[3] A. Pikovsky and J. Kurths, Synchronization: A Universal Concept in Nonlinear Sciences. Cambridge University Press, 2003.

[4] S. H. Strogatz, Sync: How Order Emerges from Chaos in the Universe, Nature, and Daily Life. Hyperion, 2004.

[5] F. Dorfler and F. Bullo, "Synchronization and transient stability in power networks and nonuniform kuramoto oscillators," SIAM Journal on Control and Optimization, vol. 50, no. 3, pp. 1616-1642, 2012.

[6] R. Wei and R. Beard, Distributed Consensus in Multi-vehicle Cooperative Control, ser. Communications and Control Engineering. Springer, 2008.
[7] V. Gazi and K. M. Passino, Swarm Stability and Optimization. Springer, 2011.

[8] J. M. González-Miranda, Synchronization and Control of Chaos: An Introduction for Scientists and Engineers. Imperial College Press, 2004.

[9] F. Lewis, H. Zhang, K. Hengster-Movric, and A. Das, Cooperative Control of Multi-Agent Systems, ser. Communications and Control Engineering. Springer, 2014.

[10] J. S. Shamma, Cooperative Control of Distributed Multi-Agent Systems. Wiley-Interscience, 2008.

[11] R. Olfati-Saber, "Flocking for multi-agent dynamic systems: algorithms and theory," Automatic Control, IEEE Transactions on, vol. 51, no. 3 , pp. 401-420, 2006.

[12] S. Dashkovskiy, D. Efimov, and E. Sontag, "Input to state stability and allied system properties," Automation and Remote Control, vol. 72, no. 8, pp. 1579-1614, 2011.

[13] D. Angeli, J. Ferrell, and E. Sontag, "Detection of multistability, bifurcations and hysteresis in a large class of biological positive-feedback systems," Proc. Natl. Acad. Sci. USA, vol. 101, pp. 1822-1827, 2004.

[14] D. Angeli and E. Sontag, "Multi-stability in monotone input/output systems," Systems\&Control Lett., vol. 51, pp. 185-202, 2004.

[15] A. Gelig, G. Leonov, and V. Yakubovich, Stability of nonlinear systems with non unique equilibrium. Moscow: Nauka, 1978, [in Russian].

[16] G.-B. Stan and R. Sepulchre, "Analysis of interconnected oscillators by dissipativity theory," IEEE Trans. Automatic Control, vol. 52, pp. 256270, 2007.

[17] M. Chaves, T. Eissing, and F. Allgower, "Bistable biological systems: A characterization through local compact input-to-state stability," IEEE Trans. Automatic Control, vol. 45, pp. 87-100, 2008.

[18] A. Rantzer, "A dual to Lyapunov's stability theorem," Syst. Control Lett., vol. 42, pp. 161-168, 2001.

[19] D. Angeli, "An almost global notion of input-to-state stability," IEEE Trans. Automatic Control, vol. 49, pp. 866-874, 2004.

[20] D. Angeli and D. Efimov, "On input-to-state stability with respect to decomposable invariant sets," in Proc. 52nd IEEE Conference on Decision and Control, Florence, 2013.

[21] D. Efimov, "Global lyapunov analysis of multistable nonlinear systems," SIAM Journal on Control and Optimization, vol. 50, no. 5, pp. 31323154, 2012.

[22] Z. Nitecki and M. Shub, "Filtrations, decompositions, and explosions," American Journal of Mathematics, vol. 97, no. 4, pp. 1029-1047, 1975.

[23] E. Egorov and A. Koronovskii, "Dynamical control in multistable systems," Technical Physics Letters, vol. 30, no. 3, pp. 186-189, 2004

[24] J. Guckenheimer and P. Holmes, "Structurally stable heteroclinic cycles," Math. Proc. Camb. Phil. Soc., vol. 103, pp. 189-192, 1988.

[25] Z. Li, Z. Duan, G. Chen, and L. Huang, "Consensus of multiagent systems and synchronization of complex networks: A unified viewpoint," Circuits and Systems I: Regular Papers, IEEE Transactions on, vol. 57, no. 1, pp. 213-224, 2010.

[26] J. Zhao, D. Hill, and T. Liu, "Global bounded synchronization of general dynamical networks with nonidentical nodes," Automatic Control, IEEE Transactions on, vol. 57, no. 10, pp. 2656-2662, 2012

[27] A. Y. Pogromsky, "A partial synchronization theorem," Chaos, vol. 18, p. 037107,2008

[28] E. Sontag and Y. Wang, "Lyapunov characterizations of input to output stability," SIAM J. Control and Optimization, vol. 39, pp. 226-249, 2001.

[29] D. Angeli and E. Sontag, "Forward completeness, unboundedness observability, and their Lyapunov characterizations," Syst. Control Lett., vol. 38, no. 4-5, pp. 209-217, 1999.

[30] A. Fradkov, B. Andrievsky, and K. Boykov, "Control of the coupled double pendulums system," Mechatronics, vol. 15, no. 10, pp. 1289$1303,2005$.

[31] A. L. Fradkov and B. Andrievsky, "Synchronization and phase relations in the motion of two-pendulum system," International Journal of NonLinear Mechanics, vol. 42, no. 6, pp. 895-901, 2007.

[32] H. Ahmed, R. Ushirobira, D. Efimov, and W. Perruquetti, "On conditions of robust synchronization for multistable systems," in Proc. European Control Conference (ECC) 2015, 2015.

[33] D. Efimov, R. Ortega, and J. Schiffer, "Iss of multistable systems with delays: application to droop-controlled inverter-based microgrids," in Proc. ACC 2015, Chicago, 2015. 